\title{
Das Oldenburger Mathe-Camp
}

\author{
Daniel Grieser und Maren Hawighorst
}

\begin{abstract}
Mathematikbegeisterte SchülerInnen aus der Region Oldenburg in Niedersachsen können seit 2014 jeden Januar auf dem dreitägigen Mathe-Camp ihrer Leidenschaft frönen. Es gibt zahlreiche ähnliche Aktivitäten in Deutschland und international, doch unsere Erfahrung zeigt, dass der Bedarf groß ist: Es könnten noch viel mehr sein. Wir wollen kurz über unser

Mathe-Camp berichten, um zu informieren und anderen Mut zu machen, ihr eigenes

Mathe-Camp ins Leben zu rufen.
\end{abstract}

\section{Entstehung}

JedeR von uns (DG und MH) hatte schon seit längerem die Idee mit sich herumgetragen, ein lokales Angebot für SchülerInnen zu schaffen, die der Schulunterricht in Mathematik nicht ausreichend fordert. An einigen Schulen gab es bereits Mathe-AGs, doch dies sollte inhaltlich darüber hinausgehen und schulübergreifend sein. Vor allem sollte sich die neue Veranstaltung über mindestens zwei Tage erstrecken.

Doch wie anfangen, wie die Lehrkapazität bereitstellen? Bei einer Schulveranstaltung kamen wir zufällig miteinander ins Gespräch und beschlossen, die Ressourcen von Uni und Schule zu bündeln: das Mathe-Camp war geboren.

\section{Durchführung}

Etwa 5o SchülerInnen der Klassen 5-13 kommen für drei Tage in einem Bildungshaus zusammen. In altersgestaffelten Gruppen (Klassen 5, 6, 7/8, 9/10, 11-13) lernen sie Mathematik, die über den Schulstoff hinausgeht. In jeder der 9o-minütigen Kurseinheiten werden sie in ein Themengebiet eingeführt und haben dann viel Zeit, es in angeleiteter Eigenarbeit anhand von Aufgaben und anderen Arbeitsmaterialien weiter zu erkunden.

Wer leitet die Gruppen? Zum einen ausgewählte fortgeschrittene Studierende der Uni Oldenburg (aus Lehramts- oder Fachstudiengang Mathematik), die neben dem systematischen einen problemlösenden Blick auf die Mathematik haben - etwa angeregt durch eigene Erfahrungen in Mathe-AGs, Wettbewerbe oder durch das Uni-Modul Mathematisches Problemlösen und Beweisen. Zum anderen LehrerInnen, die Herausforderungen jenseits des Schulunterrichts suchen.

Die Themen der Kurseinheiten reichen von Geometrie über Logik, Beweise, Gleichungen/Ungleichungen, Zahlentheorie, Kombinatorik bis zu „Universitätsmathematik“ (für die Klassen 11-13). Um die Inhalte abzustimmen, haben wir ein Curriculum erstellt, welches über die Jahre weiterentwickelt wurde.

Ergänzt wird das Programm durch gemeinsame Freizeitaktivitäten und durch einen Vortrag von einem Mathematik-Professor über ein Thema, das für alle (Klassen 5-13) etwas Spannendes bereithält. Ja, solche Themen gibt es - beispielsweise Vier-Farben-Satz oder Gitterpunkte!

\section{Aufwand und Nutzen}

Das Mathe-Camp wird erst durch viel ehrenamtliches Engagement möglich: Vorbereitung von Arbeitsmaterialien, Einwerbung von Mitteln, Organisation von Unterkunft, Programm, Abrechnung usw. Beim ersten Mal ist der Aufwand am größten, danach profitiert man von der bisherigen Arbeit, den geschaffenen Strukturen und den gemachten Erfahrungen.

Am Ende jedes Mathe-Camps sind sich jedoch immer alle einig: es hat sich gelohnt! $\mathrm{Zu}$ den positiven Effekten für die verschiedenen Beteiligten zählen insbesondere: Die SchülerInnen werden in ihrer MathematikBegeisterung ernst genommen und gefördert, sie treffen Gleichgesinnte und verbessern ihre Chancen bei Wettbewerben wie der Mathematik-Olympiade - dieser Effekt war in den letzten Jahren sehr deutlich zu sehen.

Für die Lehrenden ist das Arbeiten mit den hochmotivierten SchülerInnen eine bereichernde Erfahrung, oft nehmen sie neue Einsichten mit, mathematische und sonstige. Sie lernen andere Lehrende mit großem fachlichen Interesse kennen, und der informelle Austausch zwischen den LehrerInnen und Uni-Vertretern bereichert beide Seiten.

Die Lehramtsstudierenden erhalten einen anderen Einblick in den Umgang mit Schülern als z. B. beim Schulpraktikum. Sie werden angeregt, später selbst solche Angebote an ihrer Schule anzubieten. 


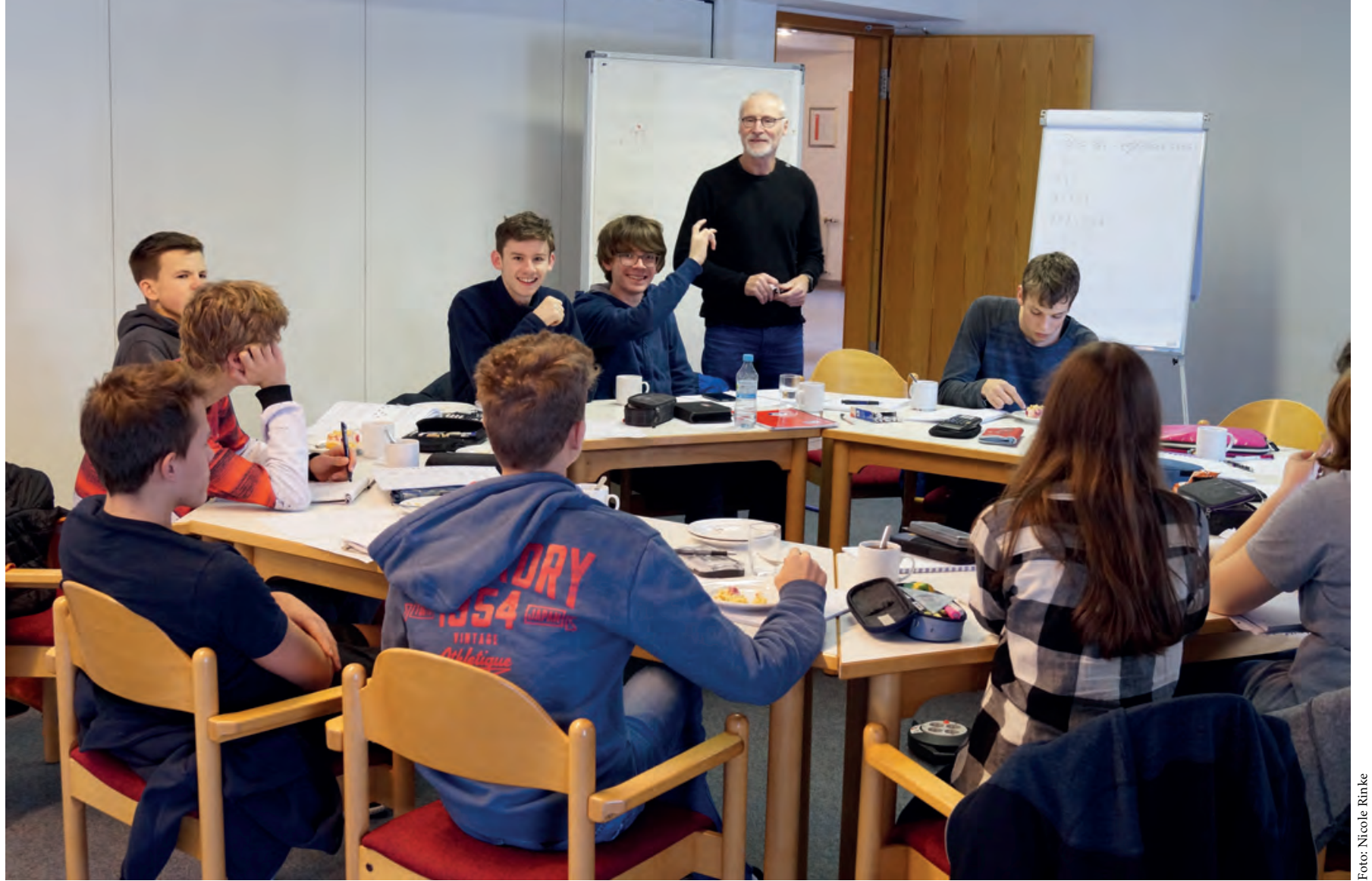

Mathe-Begeisterung beim Oldenburger Mathe-Camp

\section{Weitere Aspekte}

Jedes Jahr melden sich weitere LehrerInnen, die z. B. über die Zeitung vom Mathe-Camp erfahren haben und sich gerne beteiligen möchten. Es gibt hier eine große Bereitschaft, sich neben den Pflichten im Schulalltag zu engagieren. Nach unserer Erfahrung unterstützen Schulleiter diese Aktivitäten durch Freistellungen für die Zeit des Mathe-Camps. Es wäre aber schön, wenn das Bildungsministerium für die Organisation Entlastungsstunden gewähren würde.

Es gibt zahlreiche Stiftungen, die solche Projekte finanziell unterstützen. Das Oldenburger Mathe-Camp wurde von Anfang an von der EWE-Stiftung unterstützt.
In den USA sind viele Mathe-Camp-artige Aktivitäten über die National Association of Math Circles vernetzt: Auf der Webseite www.mathcircles.org findet man umfangreiche Informationen und Anregungen zu allen Aspekten des Themas. Nützlich ist es zum Beispiel, Materialien auszutauschen - es muss nicht jeder das Rad neu erfinden. Eine ähnliche Vernetzung wäre daher auch im deutschsprachigen Raum zu begrüßen. Vielleicht möchte jemand unter den Leserinnen und Lesern das angehen?

Wir geben auf Anfrage gerne weitere Auskunft.

Prof. Dr. Daniel Grieser, Carl von Ossietzky Universität Oldenburg, Institut für Mathematik, 26111 Oldenburg daniel.grieser@uni-oldenburg.de

Maren Hawighorst, Gymnasium Cäcilienschule Oldenburg, Haarenufer 11, 23122 Oldenburg maren.hawighorst@gmx.de

Prof. Dr. Daniel Grieser forscht und lehrt an der Carl von Ossietzky Universität Oldenburg. Er hat als Schüler das IMO-Vorbereitungstraining genossen und 2011 an der Uni Oldenburg das neue Modul Mathematisches Problemlösen und Beweisen ins Leben gerufen.

Maren Hawighorst unterrichtet Mathematik, Physik und Informatik am Gymnasium Cäcilienschule Oldenburg. Sie war viele Jahre Beraterin des Landes Niedersachsen für SchülerInnen mit besonderen Begabungen. Seit 2006 leitet sie eine schulübergreifende Mathematik-AG für die Jahrgänge 5-13. 